\title{
On Improving Voice Capacity in 802.11 Infrastructure Networks
}

\author{
Peter Clifford, Ken Duffy, Douglas J. Leith and David Malone \\ Hamilton Institute, NUI Maynooth, \\ Co. Kildare, Ireland \\ Email: \{peter.clifford, ken.duffy, doug.leith, david.malone\}@nuim.ie
}

\begin{abstract}
In this paper we consider voice calls in $802.11 \mathrm{e}$ infrastructure mode networks. We demonstrate that in standard 802.11 WLANs it is throttling of voice traffic at the access point that is the primary limitation on voice call capacity. We demonstrate that a straightforward 802.11e prioritisation strategy avoids this throttling and yields close to the theoretical maximum call capacity.
\end{abstract}

\section{INTRODUCTION}

In recent years, 802.11 wireless LANs have become pervasive. While providing wire-free connectivity at low cost, it is widely recognized that the 802.11 MAC layer requires greater flexibility and the new 802.11e standard consequently allows tuning of MAC parameters that have previously been constant. Although the 802.11e standard provides adjustable parameters within the MAC layer, the challenge is to use this flexibility to achieve enhanced network performance.

In this paper we study the behavior of infrastructure mode 802.11 networks where traffic is transmitted via an access point (AP). Our starting point is the observation that the 802.11 Carrier Sensing Multiple Access/Collision Avoidance (CSMA/CA) mechanism enforces per station fairness, i.e. each station has approximately the same number of transmission opportunities. This includes not only the wireless stations (STAs), but also the AP itself. We show that this has profound implications for network performance.

Consider an $802.11 \mathrm{~b}$ WLAN carrying $n$ two-way voice conversations. There are $n$ wireless stations each transmitting the voice of one speaker and $n$ replies transmitted by the AP1. Figure 1 shows throughput, loss and delay for both the AP and the aggregate of the wireless stations, with increasing number of voice conversations. As the number of conversations rises above 8 , the throughput achieved by the AP falls relative to that of the wireless stations. When the number of calls exceeds approximately 10, the loss-rate of the downstream AP traffic increases beyond a viable level. These results are with small buffers, so queueing delays are short and loss is the limiting factor.

We compare this behavior with the following simple capacity calculation. Table I gives the overhead budget for the

\footnotetext{
${ }^{1}$ Parameters for the voice calls are taken from [1]: $64 \mathrm{~kb} / \mathrm{s}$ on-off traffic streams where the on and off periods are distributed with mean 1.5 seconds. Periods of less than $240 \mathrm{~ms}$ are increased to $240 \mathrm{~ms}$ in length, to reproduce the minimum talk-spurt period. Traffic is two-way; the on period of an upstream call corresponds to the off period of its downstream reply.
}
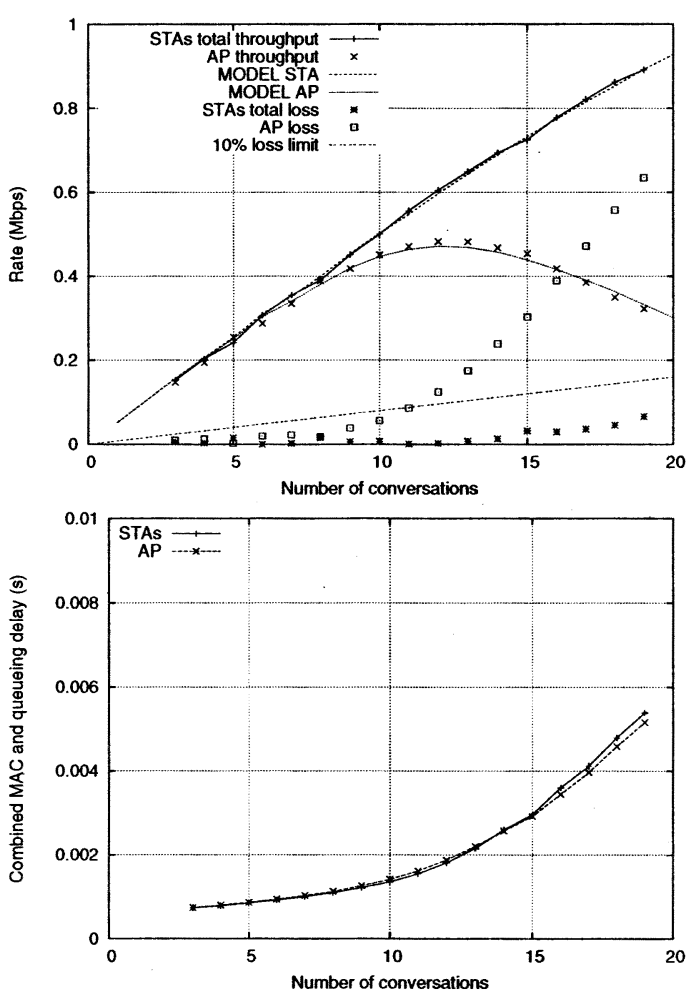

Fig. 1. Throughput and delay for competing voice calls in an infrastructure mode 802.11b WLAN ( $N S$ simulation, 802.11b WLAN, MAC parameters in Table I).

transmission of a small packet of payload 80 bytes. With overhead the transmission of an 80 bytes payload takes $651 \mu \mathrm{s}$, thus the maximum possible user throughput is approximately $80 \mathrm{~B} / 651 \mu \mathrm{s}=0.98 \mathrm{Mb} / \mathrm{s}$. That is, for $64 \mathrm{~kb} / \mathrm{s}$ voice conversations, the channel can at best support $0.98 / 0.64=15.4$ calls, which is $50 \%$ higher than the above measured capacity. The figure of 15.4 calls is over-optimistic as it neglects the idle time spent during contention window count-down as well as many other details of the channel behavior (such as packet collisions). Nevertheless, it suggests that room for improvement may well exist and it is this which is the subject of the present paper.

As voice calls are two-way, it is evident from Figure 1 that the throttling action at the AP currently acts as a primary limiting factor in voice call capacity in infrastructure networks (rather than the overall wireless bandwidth available - we 
can see that the MAC delay remains low, indicating that the channel remains relatively lightly loaded with collisions and associated exponential backoff of the 802.11 contention windows infrequent). By restoring parity between forward and reverse traffic in infrastructure networks the potential exists to increase voice call capacity. In this paper we investigate how we can use the flexibility provided by the new 802.11e MAC to avoid AP throttling in infrastructure WLANs and thereby increase network capacity.

\begin{tabular}{|l|l|}
\hline & Duration $(\mu s)$ \\
\hline Slot time, $\sigma$ & 20 \\
Propagation delay, $\delta$ & 1 \\
$C W_{\min }=32 \sigma$ & 640 \\
DIFS (AIFS=0) & 50 \\
SIFS (Short Inter Frame Space) & 10 \\
PLCP Header @ 1Mb/s & 192 \\
MAC Header 24 Bytes @1Mb/s & 17.5 \\
CRC Header 4 Bytes @ 1Mb/s & 2.9 \\
IP Header 20 Bytes @ 11Mb/s & 14.5 \\
MAC ACK 14 Bytes @ 1Mb/s & 112 \\
$E_{i}$ payload 80 Bytes @ 11Mb/s & 58.2 \\
$E_{i}$ payload 540 Bytes @ 11Mb/s & 392.73 \\
\hline
\end{tabular}

TABLE I

802.11 B MAC VALUES, BASIC RATE $1 \mathrm{MB} / \mathrm{S}$ AND DATA RATE $11 \mathrm{MB} / \mathrm{s}$.

\section{RELATED WORK}

There have been a number of previous studies of voice over 802.11 networks. Most have been concerned with measuring the voice call capacity of 802.11 networks rather than adjusting the MAC layer behavior itself. For example, in [2] a back-ofthe-envelope calculation for maximum capacity of a WLAN is presented and shown to be a useful estimate. The authors also consider, using simulation, how delay constraints and biterror rates impact the capacity of the network. Other metrics for voice capacity are used in [3] and [4].

\section{802.11 CSMA/CA}

The 802.11 MAC layer uses a CSMA/CA algorithm with binary exponential back-off to regulate access to the shared wireless channel. Briefly, on detecting the wireless medium to be idle for a period $D I F S$, each station initializes a counter to a random number selected uniformly from the interval $[0, \mathrm{CW}-$ 1]. Time is slotted and this counter is decremented for each slot that the medium is idle. An important feature is that the count-down halts when the medium becomes busy and only resumes after the medium is idle again for a period DIFS. On the counter reaching zero, the station is permitted to transmit for a time $T X O P$ on the medium (defined to be one packet in 802.11). If a collision occurs (two or more stations transmit simultaneously), $\mathrm{CW}$ is doubled and the process repeated. On a successful transmission, $\mathrm{CW}$ is reset to the value $C W_{\min }$ and a new count-down starts for the next packet. The new 802.11e MAC enables the values of DIFS (called AIFS in $802.11 \mathrm{e}), C W_{\min }$ and $T X O P$ to be set on a per class basis for each station, with a maximum of four classes per station.

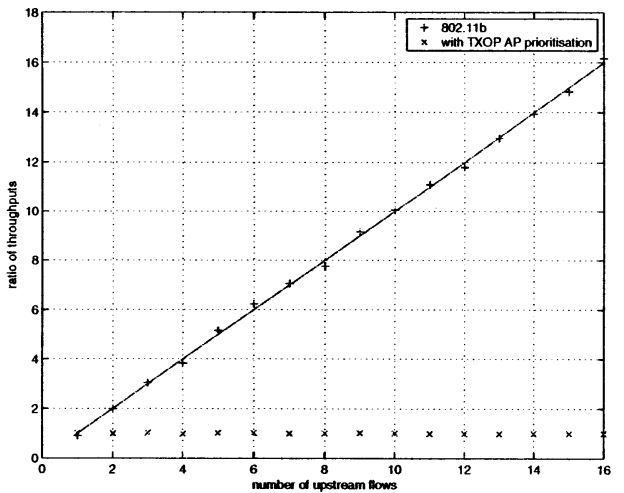

Fig. 2. Throughput of competing upload and download UDP streams vs number of streams ( $N S$ simulation, 802.11 MAC parameters in Table I).

\section{AVOIDING AP Throttling}

Our focus in this paper is on infrastructure mode networks where calls are routed through a single access point (AP). Owing to the nature of the 802.11 contention mechanism infrastructure networks behave quite differently from ad hoc peer-to-peer networks.

The 802.11 CSMA/CA mechanism provides each station with approximately the same number of transmission opportunities. This includes not only the wireless stations, but also the AP. Suppose we have $n_{u}$ wireless stations each transmitting upstream traffic and $n_{d}$ downstream flows transmitted by the AP. The $n_{u}$ wireless stations have roughly a $n_{u} /\left(n_{u}+1\right)$ share of the bandwidth while the AP has only a $1 /\left(n_{u}+1\right)$ share. It is this asymmetry that can result in the AP becoming the network bottleneck.

The validity of this argument, at least for greedy (every station always has a packet to send) flows, can be seen from Figure 2. The figure shows the ratio of the throughputs achieved by competing upstream and downstream UDP flows as the number of flows is varied (with an equal number of uploads and downloads). Evidently, the throughput ratio is equal to the number $n_{u}$ of uploads.

This simple argument leads us to propose that the AP be prioritized so as to restore parity between upstream and downstream flows. While we might prioritize downstream traffic by using an appropriate value of $C W_{\min }$ at the AP, the utility of $C W_{\min }$ is constrained by the availability of only a coarse granularity $\left(C W_{\min }\right.$ can only be varied by powers of two in 802.11e). The AIFS parameter might also be used, but is better suited to strict prioritization rather than proportional prioritization. Instead we propose that the TXOP packet bursting mechanism in $802.11 \mathrm{e}$ provides a straightforward and fine grained mechanism for prioritizing downstream traffic. Since the downstream traffic gains a $1 /\left(n_{u}+1\right)$ share of transmission opportunities, by transmitting $n_{d}$ packets (one packet to each of the $n_{d}$ downstream destination stations) at each transmission opportunity it can be immediately seen that we restore the $n_{d} /\left(n_{u}+n_{d}\right)$ fair share to the downstream traffic. This can be implemented in practice by inspecting the downstream interface queue, from which we can determine the number of distinct wireless stations to which queued 
packets are destined. This provides a direct measure of the number $n_{d}$ of active downstream flows in a manner which is both straightforward and dynamically adapts to accommodate bursty and intermittent traffic. The effectiveness of this scheme is shown in Figure 2, where it can be seen to restore fairness between the upstream and downstream flows.

Comment: Packet Burst Size. With this TXOP approach the AP transmits $n_{d}$ packets in a single burst. For $n_{d}$ large, this can result in the AP occupying the channel for a substantial consolidated period of time and this may, for example, negatively impact competing delay sensitive traffic. We can address this issue in a straightforward manner by using multiple smaller bursts instead of a single burst. When using smaller packet bursts, it is necessary to ensure a corresponding increase in the number of transmission opportunities won by the AP. This can be achieved by using a smaller value of $C W_{\min }$ for the downstream traffic class at the AP. It is shown in [5] that competing traffic classes gain transmission opportunities approximately in inverse proportion to their values of $C W_{\min }$. Let $k$ denote the ratio of the stations upstream class $C W_{\min }$ value to that of the downstream class at the AP. Scaling $k$ with the number of transmission opportunities required provides coarse (recall that in $802.11 \mathrm{e} k$ is constrained to be a power of two) prioritization of downstream flows. We then complement this with use of $T X O P$ for fine grained adjustment of the packet burst lengths, scaling $T X O P$ with $1 / k$. Hence fine grained prioritization can be achieved while avoiding unduly large packet bursts.

\section{VoICE CALlS}

While the foregoing argument provides insight and makes accurate predictions for greedy traffic flows, the situation with voice calls is more complex. We can see this immediately from Figure 1 where the upstream and downstream voice flows achieve almost equal throughput up to around 8 calls. In contrast, if the upstream/downstream flows were greedy (always have a packet to send) then the foregoing analysis indicates that with 8 calls the upstream flows would in aggregate achieve a factor of 8 greater throughput than the downstream flows.

We can understand this behavior by noting that, firstly, voice traffic is relatively low rate and so need not make use of every available transmission opportunity awarded by the 802.11 MAC. Secondly, a voice conversation involves speakers approximately taking turns at talking. That is, traffic is between pairs of speakers with the on period of one speaker roughly corresponding to the off period of the other. Both of these features mitigate the contention between the wireless stations and the AP for access to the wireless channel.

To explore this behavior further, in the next section we discuss a heterogenous finite-load analytic model suited to voice traffic modeling. We then use this, together with simulations, in Section V-B to study the impact of AP prioritization on the behavior of voice calls in infrastructure mode networks.

\section{A. Analytic Modeling}

Following the seminal paper of Bianchi [6], much of the analytic work on 802.11 MAC performance has focused on

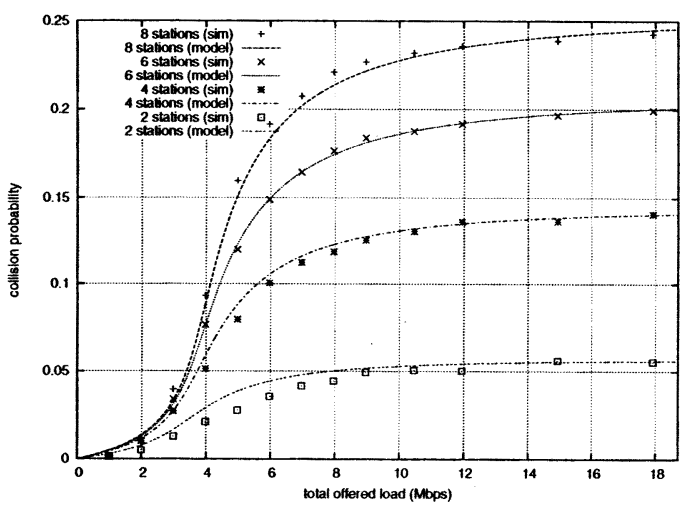

Fig. 3. Collision probability as the traffic arrival rate is varied for smaller numbers of nodes (Model and $N S$ simulation, 802.11 MAC parameters in Table I).

saturated networks where each station always has a packet to send. In particular, recent work has extended the saturation modeling approach to include multi-class $802.11 \mathrm{e}$ networks, see [5], [7] and [8]. The saturation assumption is key to these models as it enables queueing dynamics to be neglected and avoids the need for detailed modeling of traffic characteristics, making these networks particularly tractable.

Networks do not typically operate in saturated conditions. Internet applications, such as voice over IP, video and web browsing all exhibit bursty or on-off traffic characteristics. Creating an analytic model that includes fine detail of trafficarrivals and queueing behavior, as well as 802.11 MAC operation, presents a significant challenge. We introduce an 802.11 model with traffic and buffering assumptions that make it sufficiently simple to give explicit expressions for quantities of interest (throughput per station and collision probabilities), but still capture key effects of non-saturated operation. Although our traffic assumptions form only a subset of the possible arrival processes, we will see they are useful in modeling a wide range of traffic, in particular voice conversations.

Details of our analytic model are contained in the Appendix; using specified 802.11 MAC parameters and arrival rates, the model predicts transmission probability, collision probability and throughput. The predictive accuracy of the model is illustrated in Figures 4 and 5, where model predictions are compared with throughput data from $N S$ packet-level simulations, with 540 Byte payloads, as the arrival rate is varied across its range and as the number of wireless nodes is varied. The collision probabilities corresponding to Figure 4 are shown in Figure 3 (similar accuracy is obtained for the conditions used in Figure 5).

The utility of the model for modeling VoIP traffic, both in peer-to-peer and infrastructure mode networks, is demonstrated in Figure 1. It can be seen that the model makes remarkably accurate predictions.

\section{B. Voice Traffic}

As before, we consider setting the AP TXOP to be equal to the number of active downlink voice calls. Figure 6 shows throughput and delay with this scheme as the number of 


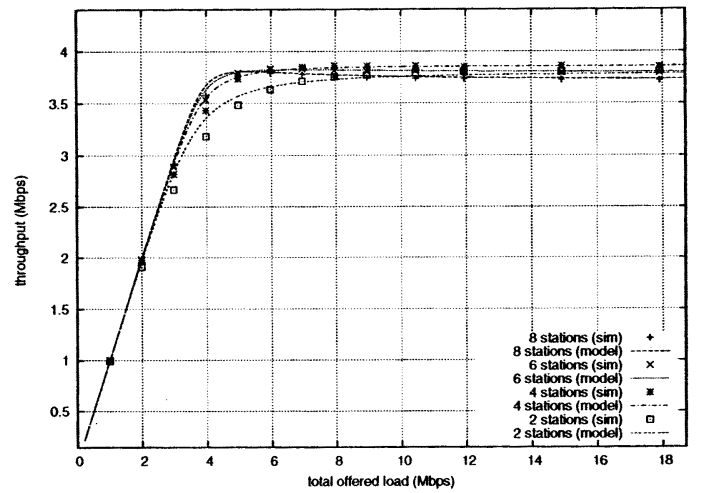

Fig. 4. Throughput as the traffic arrival rate is varied for smaller numbers of nodes. For throughput rates below those shown there is agreement between the model and simulation (Model and NS simulation, 802.11 MAC parameters in Table I).

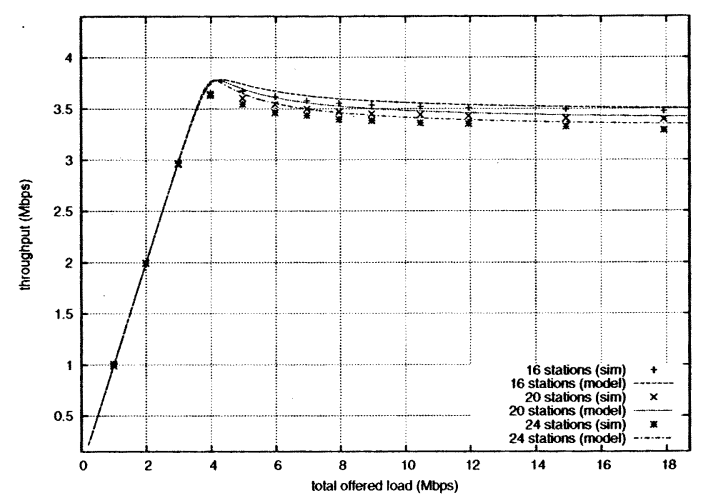

Fig. 5. Throughput as the traffic arrival rate is varied for larger numbers of nodes. For throughput rates below those shown there is agreement between the model and simulation (Model and NS simulation, 802.11 MAC parameters in Table I).

voice calls is increased. It can seen that now both the AP and wireless stations achieve similar throughputs, with the simulation and model results in good agreement. The network can now sustain approximately 15 voice calls before the delay exceeds the packet inter-arrival time of $10 \mathrm{~ms}$ and the system enters an unstable queueing regime where delays and loss rapidly increase.

The capacity is also in good agreement with the simple calculation given in the Introduction which establishes that the best case capacity is 15.4 voice calls. We can see that the measured capacity of 15 calls is remarkably close to this ideal value $^{2}$ which indicates that the scope for further performance improvement is limited.

\footnotetext{
${ }^{2}$ With $C W_{\min }=32$, we expect that the average count-down time is $C W_{\min } / 2=16$ slots or $320 \mu \mathrm{s}$. Using this value the capacity of the wireless channel falls to only 10 calls using our simple calculation. A measured capacity of 15 voice calls implies that the average count-down time is significantly less than $C W_{\min } / 2$. This can be explained by noting that when voice packets are generated by many stations statistical multiplexing of packet transmissions takes place. Hence, the average time the medium is idle is much less than the average per flow backoff time.
}
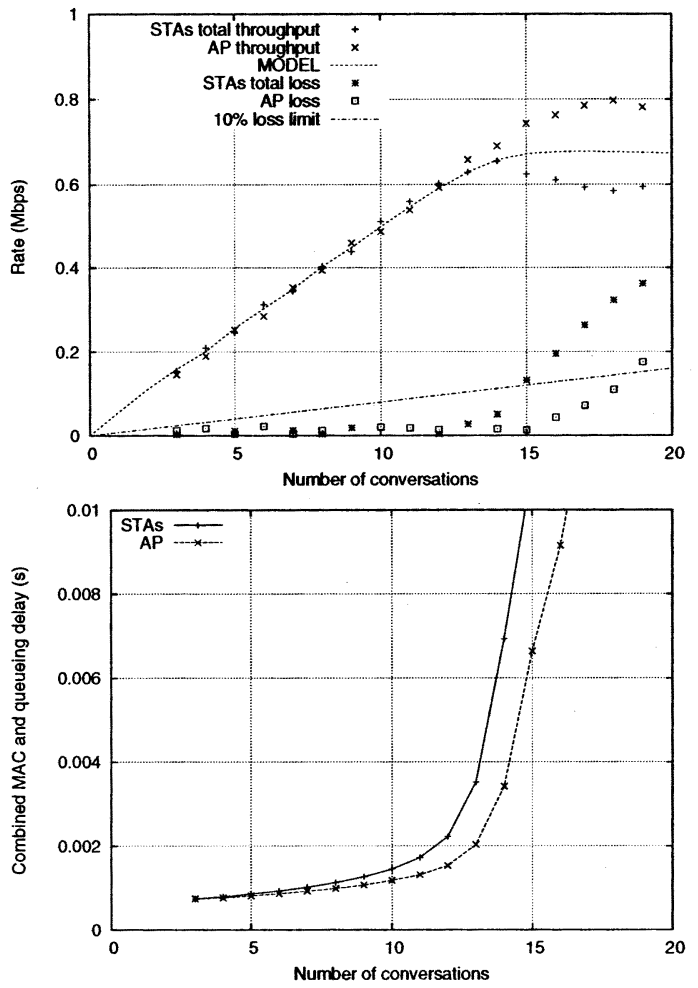

Fig. 6. Throughput and delay for competing voice with prioritization of the AP using the 802.11e TXOP.

\section{CONCLUSIONS}

In this paper our objective has been to develop a soundlybased strategy for selecting 802.11e MAC parameters in infrastructure networks carrying voice traffic. Specific contributions include the following:

- A demonstration that gross unfairness can exist between upstream and downstream flows in 802.11 infrastructure networks. This is associated with MAC level enforcement of per station fairness.

- The introduction of an new analytic model of 802.11 networks is developed that is capable of capturing the behavior of voice traffic.

- A straightforward 802.11e prioritization strategy for infrastructure networks that avoids throttling of voice flows at the AP.

The proposed 802.11e prioritization approach has the merit of being very straightforward and of imposing only a small computational burden (e.g. we use simple parameter settings and complex measurement-based adaptation is avoided). The approach is compatible with the WME subset of 802.11e that is supported by currently available hardware.

\section{ACKNOWLEDGMENTS}

This work was supported by Science Foundation Ireland grant 03/IN3/I396.

\section{APPENDIX}

Bianchi [6] presents a Markov model where each station 217

is modeled by a pair of integers $(i, k)$. The back-off stage, 
$i$, starts at 0 at the first attempt to transmit a packet and is increased by 1 every time a transmission attempt results in a collision, up to a maximum value $m$. It is reset after a successful transmission. The counter, $k$ is initially chosen uniformly between $\left[0, W_{i}-1\right]$, where typically $W_{i}=2^{i} W_{0}$ is the range of the counter and $W_{0}$ is the 802.11 parameter CWmin. While the medium is idle, the counter is decremented. Transmission is attempted when $k=0$.

We introduce new states $(0, k)_{e}$ for $k \in\left[0, W_{0}-1\right]$, representing a node which has transmitted a packet, but has none waiting. This is called postbackoff. Note that $i=0$ in all such states, because if $i>0$ then a collision has occurred, so we must have a packet awaiting transmission.

We assume that for each station there is a constant probability $1-q$ that the station's buffer has no packets awaiting transmission at the start of each counter decrement. This enables us to derive relationships between the per-station quantities: $q$, the probability of at least one packet awaiting transmission $^{3}$ at the start of a counter decrement; $m$, the maximum backoff stage; $p$, the probability of collision given the station is attempting transmission; $P$, the Markov chain's transition matrix; $b$, the chain's stationary distribution; and $\tau$, the stationary distribution's probability that the station transmits in a slot. These relationships can be solved for $p$ and $\tau$, and network throughput predicted. It is important to note that the Markov chain's evolution is not real-time, and so the estimation of throughput requires an estimate of the average state duration.

Under our assumptions, we have for $0<k<W_{i}$

$$
\begin{aligned}
0<i \leq m, \quad P[(i, k-1) \mid(i, k)] & =1, \\
P\left[(0, k-1)_{e} \mid(0, k)_{e}\right] & =1-q, \\
P\left[(0, k-1) \mid(0, k)_{e}\right] & =q .
\end{aligned}
$$

If the counter reaches 0 and a packet is queued, then we begin a transmission. We assume there is a station-dependent probability $p$ that other stations transmit at the same time, resulting in a collision. In the case of a collision we must increase the backoff stage (or discard). In the case of a successful transmission we return to backoff stage 0 and the station's buffer is empty with probability $1-q$. In the case with infinitely many retransmission attempts we need introduce no extra per-station parameters and for $0 \leq i \leq m$ and $k \geq 0$ we have

$$
\begin{aligned}
P\left[(0, k)_{e} \mid(i, 0)\right] & =\frac{(1-p)(1-q)}{W_{0}}, \\
P[(0, k) \mid(i, 0)] & =\frac{(1-p) q}{W_{0}}, \\
P[(\min (i+1, m), k) \mid(i, 0)] & =\frac{W_{\min (i+1, m)}}{W_{\operatorname{mon}}} .
\end{aligned}
$$

Naturally, these transitions could be adapted to allow discards after a certain number of transmission attempts.

\footnotetext{
${ }^{3}$ In order to move between model and simulation arrival rates, we use the following logic. When we have small buffers, the parameter $q_{i}$ is the probability that at least one packet arrives in the expected time spent per state, $E_{s}$ defined in equation (8). In simulation, the probability that at least one packet arrives during $E_{s}$ is one minus the probability that the first interpacket time is greater than $E_{s}$. Hence, when inter-packet arrival times are exponentially distributed the exponential rate $\lambda_{i}$ should be set so that $q_{i}=$ $1-\exp \left(-\lambda_{i} E_{s}\right)$, i.e. $\lambda_{i}=-\log \left(1-q_{i}\right) / E_{s}$. With $\lambda_{i}$ so chosen, the arrival rate in the model and in simulation agree. For voice conversations, in the model we use exponential distributions with mean that gives the correct CBR rate.
}

The final transitions are from the $(0,0)_{e}$ state, where postbackoff is complete, but the station's buffer is empty. In this case we remain in this state if the station's buffer remains empty. If a packet arrives we have three possibilities: successful transmission, collision or, if the medium is busy, the 802.11 MAC begins another stage- 0 backoff, now with a packet. With $P_{\text {idle }}$ denoting the probability that the medium is idle during a typical slot, the transitions from the $(0,0)_{e}$ state are:

$$
\begin{aligned}
P\left[(0,0)_{e} \mid(0,0)_{e}\right] & =1-q+\frac{q P_{\text {idle }}(1-p)}{W_{0}} \\
k>0, \quad P\left[(0, k)_{e} \mid(0,0)_{e}\right] & =\frac{q P_{\text {idle }}(1-p)}{W_{0}} \\
k \geq 0, \quad P\left[(1, k) \mid(0,0)_{e}\right] & =\frac{q P_{\text {idle }} p}{W_{1}} \\
k \geq 0, \quad P\left[(0, k) \mid(0,0)_{e}\right] & =\frac{q\left(1-P_{\text {idle }}\right)}{W_{0}} .
\end{aligned}
$$

Observe that $p$, the probability of a collision given that we are about to transmit, is the probability that at least one other station is transmitting. This is also the probability that the medium is busy if we know the station under consideration has been silent. Hence we substitute $P_{\text {idle }}=1-p$.

Given the collision probability $p$ for this station in the system and per-station parameters $q, W_{i}$ and $m$ we may solve for a stationary distribution of this Markov chain. This will enable us to determine the probability $\tau$ that this station is attempting transmission in a typical slot.

First we make observations that aid in the determination of the stationary distribution. With $b(i, k)$ and $b(0, k)_{e}$ denoting the stationary probability of being in states $(i, k)$ and $(0, k)_{e}$, as $b$ is a probability distribution we have

$$
\sum_{i=0}^{m} \sum_{k=0}^{W_{i}-1} b(i, k)+\sum_{k=0}^{W_{0}-1} b(0, k)_{e}=1 .
$$

We will write all probabilities in term of $b(0,0)_{e}$ and use the normalization in equation (1) to determine $b(0,0)_{e}$. We have the following relations. To be in the sub-chain $(1, k)$, the following must have occurred: a collision from state $(0,0)$ or an arrival to state $(0,0)_{e}$ followed by detection of an idle medium and then a collision, so that $b(1,0)=b(0,0) p+$ $b(0,0)_{e} q(1-p) p$. Neglecting packet discard, for $i>1$ we have $b(i, 0)=p^{i-1} b(1,0)$ and so

$$
\sum_{i \geq 1} b(i, 0)=\frac{b(1,0)}{1-p}=\frac{b(0,0) p+b(0,0)_{e} q(1-p) p}{1-p} .
$$

The keystone in the calculation is then the determination of $b\left(0, W_{0}-1\right)_{e}$. Transitions into $\left(0, W_{0}-1\right)_{e}$ from $(0,0)_{e}$ occur if there is an arrival, the medium is sensed idle and no collision occurs. Transitions into $\left(0, W_{0}-1\right)_{e}$ also occur from $(i, 0)$ if no collision and no arrival occurs

$$
b\left(0, W_{0}-1\right)_{e}=b(0,0)_{e} \frac{q(1-p)^{2}}{W_{0}}+\frac{(1-p)(1-q)}{W_{0}} \sum_{i \geq 0} b(i, 0)
$$

Combining equations (2) and (3) gives

$$
b\left(0, W_{0}-1\right)_{e}=b(0,0)_{e} \frac{(1-p) q(1-p q)}{W_{0}}+b(0,0) \frac{1-q}{W_{0}} .
$$

We then have for $W_{0}-1>k>0, b(0, k)_{e}=(1-q) b(0, k+$ $1)_{e}+b\left(0, W_{0}-1\right)_{e}$, with $b(0, k)_{e}$ on the left hand side replaced by $q b(0,0)_{e}$ if $k=0$. Straight forward recursion leads to 
expressions for $b(0, k)_{e}$ in terms of $b(0,0)_{e}$ and $b(0,0)$, and so we find

$$
\frac{b(0,0)_{e}}{b(0,0)}=\frac{1-q}{q}\left(\frac{1-(1-q)^{W_{0}}}{q W_{0}-(1-p)(1-p q)\left(1-(1-q)^{W_{0}}\right)}\right)
$$

Using these equations we can determine the second sum in equation (1)

$$
\sum_{k=0}^{W_{0}-1} b(0, k)_{e}=b(0,0)_{e} \frac{q W_{0}}{1-(1-q)^{W_{0}}} .
$$

The $(0, k)$ chain can then be tackled, starting with the relation

$$
b\left(0, W_{0}-1\right)=\sum_{i \geq 0} b(i, 0) \frac{(1-p) q}{W_{0}}+b(0,0)_{e} \frac{q p}{W_{0}} .
$$

Recursion leads to

$$
\begin{aligned}
\sum_{k=0}^{W_{0}-1} b(0, k) & =b(0,0)_{e}\left[\frac{q}{1-q} \frac{W_{0}+1}{2}\right. \\
& \left(\frac{q^{2} W_{0}}{1-(1-q)_{0}^{W}}+p(1-q)-q(1-p)^{2}\right) \\
& \left.+\frac{q W_{0}\left(q W_{0}+q-2\right)}{2\left(1-(1-q)_{0}^{W}\right)}+1-q\right] .
\end{aligned}
$$

Using equation(4) we can determine $b(1,0)$ in terms of $b(0,0)_{e}$ :

$$
b(1,0)=b(0,0)_{e} \frac{p q^{2}}{1-q}\left(\frac{W_{0}}{1-(1-q)_{0}^{W}}-(1-p)^{2}\right) .
$$

Finally, the normalization (1) gives

$$
\begin{aligned}
& 1 / b_{(0,0)_{e}}=(1-q)+\frac{q^{2} W_{0}\left(W_{0}+1\right)}{2\left(1-(1-q)^{W_{0}}\right)} \\
&+\frac{q\left(W_{0}+1\right)}{2(1-q)}\left(\frac{q^{2} W_{0}}{1-(1-q)^{W_{0}}}+\right. \\
&\left.p(1-q)-q(1-p)^{2}\right) \\
&+\frac{p q^{2}}{2(1-q)(1-p)}\left(\frac{W_{0}}{1-(1-q)^{W_{0}}}-(1-p)^{2}\right) \\
&\left(2 W_{0} \frac{1-p-p(2 p)^{m-1}}{1-2 p}+1\right) .
\end{aligned}
$$

The main quantity of interest is $\tau$, the probability that the station is attempting transmission. A station attempts transmission if it is in the state $(i, 0)$ (for any $i$ ) or if it is in the state $(0,0)_{e}$, a packet arrives and the medium is sensed idle. Thus $\tau=q(1-p) b(0,0)_{e}+\sum_{i \geq 0} b(i, 0)$, which reduces to

$$
\tau=b(0,0)_{e}\left(\frac{q^{2} W_{0}}{(1-p)(1-q)\left(1-(1-q)^{W_{0}}\right)}-\frac{q^{2}(1-p)}{1-q}\right),
$$

where $b(0,0)_{e}$ is given in equation (5), so that $\tau$ is expressed solely in terms of $p, q, W_{0}$ and $m$. While $q, W_{0}$ and $m$ are fixed for each station, in order to determine the collision probability, $p$, we must give a relation between the stations competing for the medium.

Consider the case where $n$ stations are present, labeled $l=1, \ldots, n$. Equation (6) gives an expression for $\tau_{l}$, the per-station transmission probability, in terms of a per-station arrival process $q_{l}$ and a per-station collision probability $p_{l}$. Observe that

$$
1-p_{l}=\prod_{j \neq l}\left(1-\tau_{j}\right), \text { for } l=1, \ldots, n
$$

that is, there is no collision for station $l$ when all other stations are not transmitting. With $n$ stations, (6) and (7) provide $2 n$ coupled non-linear equations which can be solved numerically for $p_{l}$ and $\tau_{l}$.

The length of each state in the Markov chain is not a fixed period of real time. Each state may be occupied by a successful transmission, a collision or the medium being idle. To convert between states and real time, we must calculate the expected time spent per state, which is given by

$$
\begin{aligned}
E_{s} & =\left(1-P_{t r}\right) \sigma+\sum_{i=1}^{n} P_{s: i} T_{s: i} \\
& +\sum_{r=2}^{n} \sum_{1 \leq k_{1}<\cdots<k_{r} \leq n} P_{c: k_{1} \ldots k_{r}} T_{c: k_{1} \ldots k_{r}},
\end{aligned}
$$

where $P_{s: i}=\tau_{i} \prod_{j \neq i}\left(1-\tau_{j}\right)$ is the probability station $i$ successfully transmits; $T_{s: i}$ is the time taken for a successful transmission from station $i ; P_{c: k_{1} \ldots k_{r}}=\prod_{i=1}^{r} \tau_{k_{r}} \prod_{j \neq k_{1} \ldots k_{r}}(1-$ $\left.\tau_{j}\right)$, the probability that only the stations labeled $k_{1}$ to $k_{r}$ experience a collision by attempting transmission; $T_{c: k_{1} \ldots c k_{r}}$ is the time taken for a collision from stations labeled $k_{1}$ to $k_{r} ; P_{t r}=1-\prod_{i=1}^{n}\left(1-\tau_{i}\right)$ is the probability at least one station attempts transmission; and $\sigma$ is the slot-time. For example, using the basic $802.11 \mathrm{~b}$ MAC values found in Table I with payloads $E_{i}$,

$$
\begin{aligned}
\text { Header } & =\mathrm{PLCP}+\mathrm{MAC}+\mathrm{CRC}+\mathrm{IP} \\
T_{s: i} & =E_{i}+\text { Header }+\delta+\mathrm{SIFS}+\mathrm{ACK}+\mathrm{PLCP}+\delta+\mathrm{DIFS}, \\
T_{c: i} & =E_{i}+\delta+\text { Header}+\mathrm{SIFS}+\mathrm{ACKTimeout} \\
T_{c: k_{1} \ldots k_{r}} & =\max _{i=k_{1}, \ldots, k_{r}} T_{c: i}
\end{aligned}
$$

where for 802.11b ACKTimeout is the time taken for an ACK plus PLCP plus $\delta$ plus DIFS, making $T_{s: i}=T_{c: i}$.

The normalized throughput of the system is then $S=$ $\sum_{i=1}^{n} S_{i}$, with $S_{i}=P_{s: i} E_{i} / E_{s}$ and where $E_{i}$ is the time spent transmitting payload data for source $i$. Thus in order to determine the throughput and collision probability for each station, and the overall throughput, one first solves equations (7) using equations (5) and (6). Then one uses equations (8), and the expressions for $S_{i}$ and $S$.

\section{REFERENCES}

[1] A. Markopoulou, F. Tobagi, and M. Karam, "Assessing the quality of voice communications over internet backbones," IEEE Transactions on Networking, vol. 11, pp. 747-760, October 2003.

[2] D. Hole and F. Tobagi, "Capacity of an IEEE $802.11 \mathrm{~b}$ wireless LAN supporting VoIP," in International Conference on Communications, 2004.

[3] F. Anjum, M. Elaoud, D. Famolari, A. Ghosh, R. Vaidyanathan, A. Dutta, P. Agrawal, T. Kodama, and Y. Y. Katsube, "Voice performance in WLAN networks - an experimental study," in IEEE GLOBECOM, vol. 6, pp. 3504-3508, 2003.

[4] M. Coupechoux, V. Kumar, and L. Brignol, "Voice over ieee $802.11 \mathrm{~b}$ capacity," in 16th ITC Specialist Seminar on Performance Evaluation of Wireless and Mobile Networks, 2004.

[5] R. Battiti and B. Li, "Supporting service differentiation with enhancements of the IEEE 802.11 MAC protocol: models and analysis," Tech. Rep. DIT-03-024, University of Trento, May 2003.

[6] G. Bianchi, "Performance analysis of IEEE 802.11 distributed coordination function," IEEE Journal on Selected Areas in Communications, vol. 18, pp. 535-547, March 2000.

[7] J. W. Robinson and T. S. Randhawa, "Saturation throughput analysis of IEEE 802.1 le enhanced distributed coordination function," IEEE Journal on selected areas in communications, vol. 22, pp. 917-928, June 2004.

[8] Z. Kong, D. Tsang, B. Bensaou, and D. Gao, "Performance analysis of IEEE 802.11e contention-based channel access," IEEE Journal on Selected Areas in Communications, vol. 22, pp. 2095-2106, December 2004. 